\title{
Numerical Study of Acoustic Modes in Ducted Shear Flow
}

\author{
Gregory G. Vilenski \& Sjoerd W. Rienstra \\ Department of Mathematics \& Computer Science, Eindhoven University of Technology, \\ P.O. Box 513, 5600 MB Eindhoven, The Netherlands.
}

\begin{abstract}
The propagation of small-amplitude modes in an inviscid but sheared mean flow inside a duct is studied numerically. For isentropic flow in a circular duct with zero swirl and constant mean flow density the pressure modes are described in terms of the eigenvalue problem for the Pridmore-Brown equation. Since for sufficiently high Helmholtz and wavenumbers, which are of great interest for the applications, the field equation is inherently stiff, special care is taken to insure the stability of the numerical algorithm designed to tackle this problem. The accuracy of the method is checked against the well-known analytical solution for the uniform flow. The numerical method is shown to be consistent with the analytical predictions at least for the Helmholtz numbers up to 100 and the circumferential wavenumber as large as 50, typical Mach numbers being up to 0.65 .

In order to gain further insight into the possible structure of the modal solutions and to get an independent verification of the robustness of the numerical scheme, comparison to the asymptotic solution of the problem based on the WKB method is performed. The asymptotic solution is also used as a benchmark for computations with high Helmholtz numbers, where numerical solutions of other authors are not available.

The bulk of the analysis concentrates on the influence of the wall lining. The proposed numerical procedure is adapted in order to include Ingard-Myers boundary conditions. In parallel with this, the WKB solution is used to check the numerical predictions of the typical behaviour of the axial wavenumber in the complex plane, when the wall impedance varies in the complex plane.

Numerical analysis of the problem with zero mean flow at the wall and acoustic lining shows that the use of Ingard-Myers condition in combination with an appropriate slipstream approximation instead of the actual no-slip mean flow profile gives valid results in the limit of vanishing boundary-layer thickness, although the boundary layer must be very thin in some cases.
\end{abstract}




\section{Introduction}

Normal mode analysis of small-amplitude disturbances in an annular duct with an appropriately chosen mean flow has been much used in the problems of turbomachinery noise propagation. Although an engine duct is not straight, the representation of sound in a duct by modes is very advantageous because of the clarity and the theoretical insight that it provides. The first investigations for sheared mean flow were reported in the seminal paper by Pridmore-Brown [1]. For a long time, however, it was more practical to use uniform flow (e.g., Zorumski [2]), as the numerical needs are less demanding and acoustic modes can be studied independently from the hydrodynamic modes. By using the multiple scales technique for slowly varying ducts it is possible to extend the modal approach even further (Rienstra $[3,4]$, Cooper \& Peake [5]).

Although the mean flow in the inlet duct is almost uniform with vorticity concentrated in the thin boundary layer, the mean flow of the by-pass duct is strongly sheared, sometimes with swirl, which requires more precise modelling. Since in the latter case the acoustic disturbances are not irrotational any more and are coupled with the hydrodynamic disturbances due to entropy and vorticity waves, the analysis of small amplitude modes becomes much more involved both computationally and theoretically.

A wealth of literature on the numerical analysis of this problem together with a detailed comparison of various numerical approaches can be found in Eversman [6]. Of amongst more recent numerical studies, especially with regards to swirling flows, the works by Golubev \& Atassi [7], Tam \& Auriault [8], Kousen [9], Nijboer [10], Cooper \& Peake [11] should be mentioned.

The aim of this work is to consider the problem of the propagation of small disturbances in an annular duct with sheared mean flow and wall lining from the numerical point of view. The reasons for this are as follows.

Firstly, for sufficiently high Helmholtz and circumferential wavenumbers, which are of the most interest for the applications, the field equation is inherently stiff. This means that numerical stability of the algorithms designed to tackle this problem becomes a major issue to be taken into account. For sheared mean flows without swirl considered in the present work this is due to the oscillatory character of the high frequency acoustic modes and the need to accurately resolved them numerically, although in the more general case of non-zero mean swirl the highly oscillatory behaviour of nearly-convected modes may pose an even greater problem (see, for instance, Golubev \& Atassi [7]).

As opposed to their acoustic counterparts, the hydrodynamic modes in a nonswirling mean flow generally are of non-oscillatory nature (Vilenski \& Rienstra[12]), and the high frequency is not the major concern here. For these modes the main is- 
sue is the presence of the critical layer centred on the radial position where the phase speed equals the mean flow velocity. With the exception of some very special flow conditions, hydrodynamic modes are not analytic functions in the vicinity of the critical point. As a result, their accurate numerical computation proves to be a difficult task (see, for instance, Golubev \& Atassi [7], Nijboer [10]), and more comparisons between the theoretical and numerical predictions is believed to be needed here.

Secondly, even for hard walled ducts only limited data on the properties of the spectrum of the Pridmore-Brown equation currently exists in the literature and only for relatively small values of the Helmholtz number. In particular, coverage of the properties of the hydrodynamic part of the spectrum, in our view, remains insufficient. Also, the influence of the wall lining over the spectrum structure and the range of validity of the slip-stream mean flow approximation in combination with Myers condition as a limiting model for the mean flow velocity profiles with strong near-wall shear due to no-slip condition are not fully understood.

In order to gain further insight into these issues, we will develop a robust numerical method, valid for very high Helmholtz numbers. Its validity will be verified against asymptotic results of the WKB type similar to what has been proposed by Envia [13] and Cooper \& Peake [11].

The properties of the hydrodynamic part of the spectrum for the sheared mean flow without swirl (i.e., what is described by the Pridmore-Brown equation) will be studied numerically and compared with the results of the high-frequency shortwavelength asymptotic theory recently proposed in Vilenski \& Rienstra [12]. In particular, the asymptotic analysis shows that for sheared mean flow with non-zero wall velocity and in the absence of lining the number of smooth hydrodynamic modes is finite and they are localized near the duct walls (although there exists a continuous spectrum of singular modes). This result will be confirmed numerically. In parallel with this, comparisons of the numerical predictions with the WKB solution extended to the case of soft walls will be presented. It will be used to qualitatively assess the numerical prediction of the typical behaviour of the axial wavenumber in the complex plane, when the wall impedance varies in the complex plane.

The important issues of small-disturbance propagation in a non-dissipative moving medium are the problems of the mean flow stability and causality of the modal solutions. These appear to be much more difficult than one might think on the face of it and require special investigation, Rienstra \& Tester [14], Rienstra [15,16], Brambley \& Peake [17]. Therefore, we decided to leave these issues outside the scope of the present work.

The structure of the paper is as follows. In Section 2 governing equations are derived and the main asymptotic results obtained in Vilenski \& Rienstra [12] are 


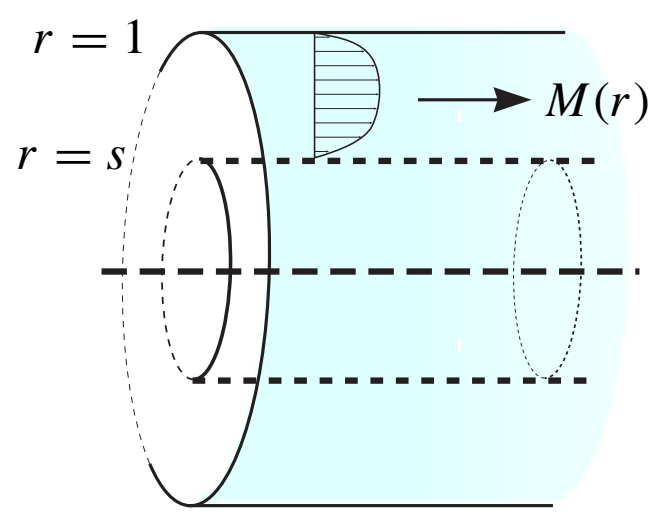

Figure 1. Flow geometry

briefly outlined, since they are needed for further comparisons with the numerical results. Section 3 describes the proposed numerical procedure for integration of the modal equations. Results of numerical analysis are presented in Section 4 which is followed by concluding remarks summarized in Section 5.

\section{Governing equations}

Consider an inviscid non-heat-conducting (i.e., isentropic) compressible perfect gas flow inside an infinitely long straight annular duct of inner radius $h$ and outer radius $d$. Let $x, r$ and $\theta$ be the axial, the radial and the circumferential coordinates, $u, v$ and $w$ the projections of the velocity vector on the coordinate axes $x, r$ and $\theta$ respectively, $\rho$ and $p$ the density and the pressure (see Figure 1). The dimensional equations for conservation of mass, radial, circumferential, axial components of momentum and energy are

$$
\begin{gathered}
\frac{\partial \rho}{\partial t}+\frac{1}{r} \frac{\partial(r \rho v)}{\partial r}+\frac{1}{r} \frac{\partial(\rho w)}{\partial \theta}+\frac{\partial(\rho u)}{\partial x}= \\
\frac{\partial v}{\partial t}+v \frac{\partial v}{\partial r}+\frac{w}{r} \frac{\partial v}{\partial \theta}+u \frac{\partial v}{\partial x}-\frac{w^{2}}{r}=-\frac{1}{\rho} \frac{\partial p}{\partial r} \\
\frac{\partial w}{\partial t}+v \frac{\partial w}{\partial r}+\frac{w}{r} \frac{\partial w}{\partial \theta}+u \frac{\partial w}{\partial x}+\frac{v w}{r}=-\frac{1}{r \rho} \frac{\partial p}{\partial \theta} \\
\frac{\partial u}{\partial t}+v \frac{\partial u}{\partial r}+\frac{w}{r} \frac{\partial u}{\partial \theta}+u \frac{\partial u}{\partial x}=-\frac{1}{\rho} \frac{\partial p}{\partial x} \\
\frac{\partial p}{\partial t}+v \frac{\partial p}{\partial r}+\frac{w}{r} \frac{\partial p}{\partial \theta}+u \frac{\partial p}{\partial x}+\gamma p\left(\frac{1}{r} \frac{\partial(r v)}{\partial r}+\frac{1}{r} \frac{\partial w}{\partial \theta}+\frac{\partial u}{\partial x}\right)=0
\end{gathered}
$$

Here $t$ is time, $\gamma=c_{p} / c_{v}$ is the ratio of specific heat capacities at constant pressure and constant volume, respectively. The pressure, the density and the absolute temperature $T$ satisfy the equation of state $p=R \rho T, R=c_{p}-c_{v}$. Assume that the total flow field is the sum of a mean base flow and small-amplitude unsteady 
perturbations

$$
(u, v, w, \rho, p)=(\bar{u}, \bar{v}, \bar{w}, \bar{\rho}, \bar{p})+(\widetilde{u}, \widetilde{v}, \widetilde{w}, \widetilde{\rho}, \widetilde{p}) .
$$

If the mean flow is independent of $x, \theta, t$ and its radial velocity is zero, then the following well known mean flow solution (see, for instance, Tam \& Auriault [8])

$$
\bar{u}=\bar{u}(r), \quad \bar{v}=0, \quad \bar{w}=\bar{w}(r), \quad \bar{\rho}=\bar{\rho}(r), \quad \bar{p}=p_{d}-\int_{r}^{d} \bar{\rho}(\xi) \frac{\bar{w}^{2}(\xi)}{\xi} \mathrm{d} \xi
$$

can be taken in order to obtain linearized Euler equations for the small-amplitude disturbance field. This mean flow is characterized by the swirl $\Omega_{x}=\mathrm{d} \bar{w} / \mathrm{d} r+\bar{w} / r$ and the circumferential vorticity (shear) $\Omega_{\theta}=-\mathrm{d} \bar{u} / \mathrm{d} r$. Here $p_{d}=\bar{p}(d)$ is a given constant.

The resulting small-amplitude disturbances are sought in the form

$$
(\widetilde{u}, \widetilde{v}, \widetilde{w}, \widetilde{\rho}, \widetilde{p})=(U, V, W, R, P) \exp (-\mathrm{i} \omega t+\mathrm{i} k x+\mathrm{i} m \theta),
$$

where $\omega$ is the excitation frequency, and $k$ and $m$ are the axial and circumferential wavenumbers, respectively. The amplitudes $(U, V, W, R, P)$ are unknown functions of $r$. They satisfy the system of equations obtained in the work by Nijboer [10]

$$
\begin{gathered}
\mathrm{i} \lambda P^{\prime}+\mathrm{i} B P-A V \bar{\rho}=0, \\
\frac{1}{r} \frac{\mathrm{d}(r V)}{\mathrm{d} r}+\frac{\mathrm{i} \Omega}{\bar{\rho} \lambda} P+C V=0 .
\end{gathered}
$$

Here the prime stands for the derivative with respect to $r$,

$$
\begin{gathered}
A=\lambda^{2}+\frac{\bar{w}^{4}}{\bar{c}^{2} r^{2}}-\frac{\bar{w}^{2}}{r} \frac{\bar{\rho}^{\prime}}{\bar{\rho}}-\frac{2 \bar{w}}{r}\left(\bar{w}^{\prime}+\frac{\bar{w}}{r}\right), \quad B=\frac{2 m \bar{w}}{r^{2}}-\frac{\lambda \bar{w}^{2}}{\bar{c}^{2} r}, \\
C=\frac{\bar{w}^{2}}{\bar{c}^{2} r}-\frac{1}{\lambda}\left(\frac{m}{r}\left(\bar{w}^{\prime}+\frac{\bar{w}}{r}\right)+k \bar{u}^{\prime}\right), \quad \Omega=\frac{\lambda^{2}}{\bar{c}^{2}}-k^{2}-\frac{m^{2}}{r^{2}}, \quad \bar{c}^{2}=\frac{\gamma \bar{p}}{\bar{\rho}}, \\
\lambda=-\omega+k \bar{u}+m \bar{w} / r .
\end{gathered}
$$

Elimination of $V$ leads to a single differential equation for the pressure amplitude $P$

$P^{\prime \prime}+\left(\frac{\lambda+B r}{\lambda r}+C+\frac{\bar{\rho} A}{\lambda} \frac{\mathrm{d}}{\mathrm{d} r}\left(\frac{\lambda}{\bar{\rho} A}\right)\right) P^{\prime}+\left(\frac{A \Omega}{\lambda^{2}}+\frac{\bar{\rho} A}{\lambda r} \frac{\mathrm{d}}{\mathrm{d} r}\left(\frac{B r}{\bar{\rho} A}\right)+\frac{B C}{\lambda}\right) P=0$.

In order to state boundary conditions for this equation, assume that the duct walls are treated with locally reacting lining with complex specific impedances $Z_{h}$ and $Z_{d}$ at $r=h$ and $r=d$, respectively. According to Ingard [18] and Myers [19], the following relations must be satisfied on the duct walls to incorporate the effects of a vanishing boundary layer

$$
-\mathrm{i} \omega \widetilde{v}_{n}=\left(-\mathrm{i} \omega+\bar{u} \frac{\partial}{\partial x}+\frac{\bar{w}}{r} \frac{\partial}{\partial \theta}\right) \frac{\tilde{p}}{Z},
$$


where $\widetilde{v}_{n}$ is the projection of the perturbation velocity on the outwardly directed normal to the duct wall $\left(\widetilde{v}_{n}=\widetilde{v}\right.$ at $r=d$ and $\widetilde{v}_{n}=-\widetilde{v}$ at $\left.r=h\right), Z=Z_{d}$ or $Z=Z_{h}$ at $r=d$ or $r=h$, respectively. Substitution of (8) in (12) together with (9) gives the required boundary conditions for the equation (11)

$$
\begin{aligned}
& P^{\prime}+\left(\frac{B}{\lambda}+\frac{\mathrm{i} \bar{\rho} A}{\omega Z_{h}}\right) P=0 \text { at } r=h, \\
& P^{\prime}+\left(\frac{B}{\lambda}-\frac{\mathrm{i} \bar{\rho} A}{\omega Z_{d}}\right) P=0 \text { at } r=d .
\end{aligned}
$$

The hard wall boundary condition on either of the walls is recovered in the limit $Z_{h}$ or $Z_{d} \rightarrow \infty$.

In the eigenvalue problem $(11,13,14)$ the excitation frequency $\omega$ and the circumferential wavenumber $m$ are given parameters while the axial wavenumber $k$ is the unknown spectral variable.

In the special case of zero mean circumferential velocity $\bar{w}=0$ and constant mean flow density $\bar{\rho}$ (and hence the pressure) (11) reduces to a form of Pridmore-Brown equation

$$
P^{\prime \prime}+\beta(r) P^{\prime}+\gamma(r) P=0
$$

where

$$
\beta(r)=\frac{1}{r}+\frac{2 k \bar{u}^{\prime}}{\omega-k \bar{u}}, \quad \gamma(r)=\frac{(\omega-k \bar{u})^{2}}{c_{0}^{2}}-k^{2}-\frac{m^{2}}{r^{2}} \quad \text { and } \quad c_{0}^{2}=\frac{\gamma p_{d}}{\bar{\rho}}
$$

In this case the boundary conditions are

$$
\begin{aligned}
& P^{\prime}+\frac{\mathrm{i} \bar{\rho}(\omega-k \bar{u})^{2}}{\omega Z_{h}} P=0 \text { at } r=h \\
& P^{\prime}-\frac{\mathrm{i} \bar{\rho}(\omega-k \bar{u})^{2}}{\omega Z_{d}} P=0 \text { at } r=d .
\end{aligned}
$$

The present work studies numerically the solutions of field equation (15) with various versions of conditions $(16,17)$. The relevant numerical procedure, which has also been used to integrate the generic problem $(11,13,14)$, is described in the next Section. However, prior to proceeding to the numerical analysis, we briefly summarize here the main analytic results for the eigenvalue problem $(15,16,17)$ reported in Vilenski \& Rienstra [12]. 


\subsection{Summary of asymptotic results}

\subsubsection{Acoustic modes}

Introduce the non-dimensional quantities

$$
\begin{gathered}
\tilde{\omega}=\frac{\omega d}{c_{0}}, \quad \tilde{k}=k d, \quad z_{h}=Z_{h} /\left(\bar{\rho} c_{0}\right), \quad z_{d}=Z_{d} /\left(\bar{\rho} c_{0}\right), \\
M=\bar{u} / c_{0}, \quad M_{h}=\bar{u}(h) / c_{0}, \quad M_{d}=\bar{u}(d) / c_{0}, \quad s=h / d,
\end{gathered}
$$

and let, for the sake of simplicity, the inner wall be hard, i.e., $Z_{h}=\infty$, the pressure amplitude $P$ be scaled by $\bar{\rho} c_{0}^{2}$, time by $d / c_{0}$, velocities by the sound speed $c_{0}, r$ and other distances by $d$. If the following short-wavelength approximation $1 \ll \widetilde{\omega} \approx$ $|m| \ll|\widetilde{k}|$ as $\widetilde{k} \rightarrow \infty$ is adopted, then, according to Vilenski \& Rienstra [12], the eigenvalue system $(15,16,17)$ becomes analytically treatable in the following three limiting cases.

Quasi-hard-wall limit. The asymptotic expansion of the axial wavenumber $\tilde{k}$ when the walls of the duct are hard is given by the series

$$
\operatorname{Re}(\widetilde{k}) \equiv \widetilde{k}_{r}^{0}=-\frac{\kappa_{0}}{\kappa_{1}}+\ldots, \quad \operatorname{Im}(\widetilde{k}) \equiv \widetilde{k}_{i}^{0}=\frac{\pi n}{\kappa_{1}}-\frac{\kappa_{-1}}{\pi n}+\ldots,
$$

where the integer $n$ is a large parameter, used to number the eigenvalues and the corresponding modal solutions, dots stand for the higher-order terms,

$$
\begin{gathered}
\kappa_{1}=\int_{s}^{1} \sqrt{1-M^{2}} \mathrm{~d} r, \quad \kappa_{0}=\int_{s}^{1} \frac{\tilde{\omega} M}{\sqrt{1-M^{2}}} \mathrm{~d} r \\
\kappa_{-1}=\frac{1}{2} \int_{s}^{1}\left\{\frac{\tilde{\omega}^{2}}{1-M^{2}}-\frac{m^{2}}{r^{2}}-\frac{2 M^{\prime 2}}{M^{2}}+\frac{M^{\prime \prime}+M^{\prime} / r}{M}+\frac{1}{4 r^{2}}\right\} \frac{\mathrm{d} r}{\sqrt{1-M^{2}}} .
\end{gathered}
$$

If the absolute value of the outer wall impedance $z_{d}=R+\mathrm{i} X$ is sufficiently large, i.e., $\left|z_{d}\right| \gg \zeta_{1} \pi n / \kappa_{1} \gg 1$, where

$$
\zeta_{1}=\frac{\widetilde{\omega}^{-1} M_{d}^{2}}{\sqrt{1-M_{d}^{2}}}
$$

then the main-order correction due to the lining results in the expression

$$
\widetilde{k} \equiv \widetilde{k}_{r}+\mathrm{i} \widetilde{k}_{i}=\left(\frac{\pi n i}{\kappa_{1}}-\frac{\kappa_{0}}{\kappa_{1}}-\frac{\mathrm{i} \kappa_{-1}}{\pi n} \ldots\right)-\frac{1}{z_{d}}\left(\frac{\zeta_{1} \pi n}{\kappa_{1}^{2}}+\ldots\right),
$$

which on elimination of the imaginary part of the impedance $X$ shows that the real $\widetilde{k}_{r}$ and the imaginary $\widetilde{k}_{i}$ parts of the axial wavenumber $\widetilde{k}$ follow the circle

$$
\left(\widetilde{k}_{r}+\frac{\kappa_{0}}{\kappa_{1}}+\frac{\zeta_{1} \pi n}{2 R \kappa_{1}^{2}}\right)^{2}+\left(\widetilde{k}_{i}-\frac{\pi n}{\kappa_{1}}+\frac{\kappa_{-1}}{\pi n}\right)^{2}=\left(\frac{\zeta_{1} \pi n}{2 R \kappa_{1}^{2}}\right)^{2}
$$


if resistance $R$ is fixed and reactance $X$ is allowed to vary. The corresponding hard-wall wavenumber lies inside this circle. The circle's radius decreases with the damping $R$ and the frequency $\omega$, but grows with the Mach number $M$ and the index $n$. The centre of the circle is shifted to the left from the position of the hardwall eigenvalue $\widetilde{k}^{0}$ by the amount of its radius for positive $n$ and to the right - for negative $n$. The neighbouring circles are shifted from one another in the vertical direction by the amount $\pi / \kappa_{1}+\mathcal{O}\left(1 / n^{2}\right)$ which depends mainly on the Mach number distribution and the hub-to-tip ratio $s$.

Limit of small resistance. Let $\mu=\zeta_{1} \pi n / \kappa_{1}, v=\zeta_{1} \kappa_{0} / \kappa_{1}$ and assume now that $|\mu| \sim|X|$, whereas resistance $R$ is small, i.e., $|\mu| \gg R$. Then, to main order, the real and imaginary parts of the axial wavenumber satisfy either of the following relations

$$
\begin{aligned}
& \widetilde{k}_{r}-\widetilde{k}_{r}^{0}=\frac{-R \cos \left(2 \kappa_{1}\left(\widetilde{k}_{i}-\widetilde{k}_{i}^{0}\right)\right)+v \sin \left(2 \kappa_{1}\left(\widetilde{k}_{i}-\widetilde{k}_{i}^{0}\right)\right)-R}{2 \mu \kappa_{1}}, \text { if } X / \mu>0, \\
& \widetilde{k}_{r}-\widetilde{k}_{r}^{0}=\frac{-R \cos \left(2 \kappa_{1}\left(\widetilde{k}_{i}-\widetilde{k}_{i}^{0}\right)\right)-v \sin \left(2 \kappa_{1}\left(\widetilde{k}_{i}-\widetilde{k}_{i}^{0}\right)\right)+R}{2 \mu \kappa_{1}}, \text { if } X / \mu<0 .
\end{aligned}
$$

Hence, when resistance $R$ is kept fixed while reactance $X$ varies, the eigenvalue $\widetilde{k}$ with a sufficiently large index $n$ must move along the sinusoidal line. The zeros of this line coincide with the successive hard-wall eigenvalues $\widetilde{k}^{0}=\widetilde{k}_{r}^{0}+\widetilde{k}_{i}^{0}$. For instance, if for some $n>0$ (or $n<0$ ) the corresponding eigenvalue $\widetilde{k}^{0}(n)$ is taken as a starting point at $X=+\infty$ and $X$ is allowed to gradually vary till $X=$ $-\infty$, then moving along the line (21) (or (22), respectively) the eigenvalue $\widetilde{k}$ will terminate at the point $\widetilde{k}^{0}(n+1)$ (or $\widetilde{k}^{0}(n-1)$, respectively). The amplitude of these sinusoidal lines decays as $1 / n$ as $n \rightarrow \infty$.

Surface modes. Two asymptotic solutions considered so far either correspond to the limit $1 \ll \zeta_{1} \pi n / \kappa_{1} \ll R$ or to its opposite $\zeta_{1} \pi n / \kappa_{1} \gg R$. Further analytical treatment of the generic dispersion relation which follows from the WKB analysis is not straightforward, and the corresponding numerical analysis is preferable here. The exception is the case of large $|X|$ whereby two branches of acoustic surface modes can be identified. For the first time, these were found numerically in Rienstra [20] in the uniform mean flow circular duct case. For a ducted flow with shear the related main-order solution is

$$
\widetilde{k}=-\mathrm{i} z_{d} / \zeta_{1}+\ldots \text { if } X \rightarrow-\infty \text { and } \widetilde{k}=\mathrm{i} z_{d} / \zeta_{1}+\ldots \text { if } X \rightarrow+\infty
$$

If the wall Mach number $M_{d}=0$, formulas (23) do not apply. This situation is studied numerically in what follows. 


\subsubsection{Hydrodynamic modes in the absence of lining}

The occurrence of a hydrodynamic mode means that at some critical point $r=\tilde{r}$ inside the duct the mode's phase speed is equal to the local mean flow speed, i.e., $\widetilde{\omega}-\widetilde{k} M(\widetilde{r})=0$. If the following analogue of the inflection point theorem

$$
\left(\frac{M^{\prime \prime}(\widetilde{r})}{M^{\prime}(\widetilde{r})}-\frac{1}{\widetilde{r}}\right)\left(\widetilde{k}^{2}+\frac{m^{2}}{\widetilde{r}^{2}}\right)+\frac{2 m^{2}}{\widetilde{r}^{3}}=0
$$

holds, there may exist a hydrodynamic mode which is smooth at $\widetilde{r}$. If otherwise, this mode develops the logarithmic singularity in the axial perturbation velocity. The analytical study of Vilenski \& Rienstra [12] shows that in the high-frequency short-wavelength limit smooth hydrodynamic modes are such that $\widetilde{r}$ is located on the walls of the duct, provided the Mach number does not vanish on the walls and its derivatives remain $\mathcal{O}(1)$ near $\widetilde{r}$. These hydrodynamic modes have maximum/minimum on the wall and decay exponentially away from it. Note however, that this conclusion may not be true if the wavelengths are not large. Apart from these modes, there is also a continuous spectrum of singular hydrodynamic modes which always feature a discontinuous third derivative, irrespective of the condition (24).

In the presence of a strongly inhomogeneous shear layer, such as, say, a viscous boundary-layer near the wall, the existence (or absence) of hydrodynamic modes, to the authors' knowledge, has not been established analytically for the eigenvalue problem $(15,16,17)$. Numerical experiments aiming to shed some extra light on the issue are presented in what follows.

\section{Numerical procedure}

The eigenvalue problem $(11,13,14)$ was solved numerically using the following method. The field equation (11) was first rewritten in the form of a system of two first-order ordinary differential equations

$$
u=P^{\prime}, \quad u^{\prime}=-\beta(r) u-\gamma(r) P
$$

where $\beta(r)$ and $\gamma(r)$ are the coefficients before $P^{\prime}$ and $P$ in equation (11). For zero circumferential mean flow velocity they are identical to $\beta(r)$ and $\gamma(r)$ appearing in the Pridmore-Brown equation (15). To reduce the amount of computations, these coefficients were rewritten as follows

$\beta(r)=\frac{B}{\lambda}+\Pi+\frac{1}{\lambda} \frac{\mathrm{d} \lambda}{\mathrm{d} r}, \quad \gamma(r)=\frac{A \Omega}{\lambda^{2}}+\frac{1}{\lambda} \frac{\mathrm{d} B}{\mathrm{~d} r}+\frac{B \Pi}{\lambda}, \quad \Pi=\frac{1}{r}-\frac{1}{\bar{\rho} A} \frac{\mathrm{d}(\bar{\rho} A)}{\mathrm{d} r}+C$, 
so that their common part $\Pi$ could be computed only once per run. System (25) was approximated by the following implicit finite-difference (backward Euler) scheme

$$
\frac{P^{j+1}-P^{j}}{\delta}=u^{j+1}, \quad \frac{u^{j+1}-u^{j}}{\delta}=-\beta^{j+1} u^{j+1}-\gamma^{j+1} P^{j+1},
$$

for $j=0,1,2,3, \ldots N-1$, where $\delta=(d-h) / N, r^{j}=h+j \delta, \beta^{j}=\beta\left(r_{j}\right)$ and $N$ is a sufficiently large integer. Since system (26) is linear, it may be solved for the unknowns $u^{j+1}$ and $P^{j+1}$ as follows.

$$
P^{j+1}=\frac{\left(1+\delta \beta^{j+1}\right) P^{j}+\delta u^{j}}{1+\delta \beta^{j+1}+\delta^{2} \gamma^{j+1}}, \quad u^{j+1}=\frac{P^{j+1}-P^{j}}{\delta},
$$

for $j=0,1,2,3, \ldots N-1$. Formulas (27) were supplemented with the initial conditions at $r=r^{0}=h$

$$
P^{0}=1, \quad u^{0}=-\left(\frac{B}{\lambda}+\frac{\mathrm{i} \bar{\rho} A}{\omega Z_{h}}\right) P^{0}
$$

and were used together with (28) to compute for a given initial guess of the spectral parameter $k=k_{n}$ the functions $u^{j+1}$ and $P^{j+1}$ and the value

$$
I\left(k_{n}\right)=\frac{1}{Q}\left[u^{N}+\left(\frac{B}{\lambda}-\frac{\mathrm{i} \bar{\rho} A}{\omega Z_{d}}\right) P^{N}\right], \quad Q=\int_{h}^{d}|P| \mathrm{d} r .
$$

Here $I\left(k_{n}\right)$ measures the error in the boundary condition on the wall $r=r^{N}=d$, the role of the parameter $Q$ is equivalent to the renormalisation of the solution after each run in $r$. Generally, it was not necessary unless very high frequencies $\omega$ and circumferential wavenumbers $m$ were used (say, for Helmholtz number equal to 80 and $m=50$ ). Since normally $I\left(k_{n}\right) \neq 0$ for an arbitrary guess value $k_{n}$, global Newtonian iterations in $k$ were needed to insure the equality $I(k)=0$. The following formula

$$
k_{n+1}=k_{n}-I\left(k_{n}\right) / I^{\prime}\left(k_{n}\right)
$$

was used to update the value of the spectral parameter $k$. The amount of computations in (30) could be reduced in comparison with the classical Newton's method if the derivative $I^{\prime}\left(k_{n}\right)$ was approximated by the finite difference

$$
I^{\prime}\left(k_{n}\right) \approx \frac{I\left(k_{n}\right)-I\left(k_{n-1}\right)}{k_{n}-k_{n-1}}
$$

(modified Newton's method). The iteration process was continued until the difference between the two successive values of $I\left(k_{n}\right)$ became smaller than a given threshold value.

An alternative approach with the initial conditions posed on the outer wall $r=$ $r^{N}=d$, formulas (27) employed in the reverse order in $j$ and the iteration procedure organized in order to satisfy the boundary condition on the inner wall $r=$ 
Table 1

WKB approximation versus numerical solution.

\begin{tabular}{ccc}
$\tilde{k}(\mathrm{WKB})$ & $\tilde{k}$ (num.) & \multicolumn{1}{c}{ error \% } \\
\hline$-49.23+\mathrm{i} \cdot 23.60$ & $-49.17+\mathrm{i} \cdot 23.78$ & $-0.06-\mathrm{i} \cdot 0.18$ \\
$-49.34+\mathrm{i} \cdot 46.67$ & $-49.32+\mathrm{i} \cdot 46.71$ & $-0.02-\mathrm{i} \cdot 0.04$ \\
$-49.41+\mathrm{i} \cdot 62.96$ & $-49.41+\mathrm{i} \cdot 62.96$ & $0.00-\mathrm{i} \cdot 0.00$ \\
$-49.46+\mathrm{i} \cdot 76.88$ & $-49.47+\mathrm{i} \cdot 76.87$ & $0.01-\mathrm{i} \cdot 0.01$ \\
$-49.50+\mathrm{i} \cdot 89.53$ & $-49.52+\mathrm{i} \cdot 89.51$ & $0.02+\mathrm{i} \cdot 0.02$
\end{tabular}

$r^{0}=h$ was also used. Although it did not have any effect on the acoustic part of the spectrum, in the case of Pridmore-Brown equation it was found more advantageous to march from the inner wall towards the outer wall when computing the hydrodynamic mode localized near the outer wall and to reverse the direction of marching for the hydrodynamic mode localized near the inner wall.

Since the present shooting method crucially depends on the solution of an initialvalue problem for the equations (25), some other marching algorithms were explored. The main idea here was to use higher-order approximations, in order to achieve better computational efficiency. As explicit multistep methods cannot be A-stable (see, for instance, Miranker [21]), only implicit schemes were considered. More specifically, for a given ordinary differential equation, say,

$$
y^{\prime}(x)=f(x, y)
$$

with $x$ and $y(x)$ being its independent and dependent variables, respectively, the implicit two-stage fourth-order Runge-Kutta method (Butcher [22])

$$
k_{i}=f\left(x_{j}+c_{i} \delta, y_{j}+\sum_{s=1}^{2} a_{i s} k_{s}\right), \quad y_{j+1}=y_{j}+\delta \sum_{s=1}^{2} b_{s} k_{s},
$$

with

$$
\begin{gathered}
c_{1}=\frac{1}{2}-\frac{\sqrt{3}}{6}, c_{2}=\frac{1}{2}+\frac{\sqrt{3}}{6}, b_{1}=b_{2}=\frac{1}{2}, \\
a_{11}=a_{22}=\frac{1}{4}, a_{12}=\frac{1}{4}-\frac{\sqrt{3}}{6}, a_{21}=\frac{1}{4}+\frac{\sqrt{3}}{6},
\end{gathered}
$$

and the following two implicit second-order accurate schemes considered in Fornberg \& Driscoll [23] for integration of stiff initial-value problems were used

$$
y_{j+1}-y_{j}=\frac{1}{2} \delta\left(y_{j+1}^{\prime}+y_{j}^{\prime}\right) \text { and } y_{j+1}-y_{j}=\frac{1}{2} \delta\left(\frac{3}{2} y_{j+1}^{\prime}+\frac{1}{2} y_{j-1}^{\prime}\right) .
$$

Here $\delta=x_{j+1}-x_{j}$ is the distance between the successive mesh points $x_{j}$ and $x_{j+1}$. The first of these two approximations is the classical Crank-Nicolson (trapezoidal) scheme, the second scheme is its more stable modification suggested in Chan \& Kerkhoven [24]. 
Although implicit Runge-Kutta methods require the solution of nonlinear equations for $k_{i}$ at each step which normally limits their practical use, this complication does not arise here. In the present problem the function $f(x, y)$ is linear, and the unknown coefficients $k_{i}$ can be easily found analytically.

Of these three schemes the method which allows for a larger stable step size $\delta$ for the stiff problem under consideration was preferred. Our computations showed that all three approximations work well for low and moderate frequency acoustic modes. In cases of very large Helmholtz numbers, however, $(\widetilde{\omega} \approx 80$ and above $)$ Newton's method failed to converge sometimes with these schemes for the cut-on wavenumber with the largest absolute value.

This was not the case with the above described implicit first-order scheme (backward Euler). It is believed that this was due to the fact that the backward Euler scheme has less severe stability restrictions on the values of $\omega$ and $k$ for a given step size.

As it has been already mentioned in the Introduction, another subtle computational issue associated with system (25) is due to the existence of the critical point, say $r_{*}$, where the mean flow velocity equals to the local phase speed of the perturbation solution. At this point the coefficient $\beta(r)$ goes to infinity and the right-hand side of (25) is singular. As is known (see, for instance, Deuflhard [25]), the application of "standard" software for such initial-value problems normally results in exponential overflow and is no longer possible. Thus, special consideration of the analytical properties of the solution was needed in the construction of the numerical algorithm.

In the case of a smooth non-swirling mean flow with shear considered in this paper the function $\beta(r)$ behaves like $-\kappa /\left(r-r_{*}\right)$ as $r$ goes to $r_{*}$, where $\kappa$ is a given constant. It follows from (25) that if the continuously differentiable solution at the critical point exists it must be such that

$$
u\left(r_{*}\right) \equiv P^{\prime}\left(r_{*}\right)=0 \text { and } u^{\prime}\left(r_{*}\right)=\kappa u^{\prime}\left(r_{*}\right)-\gamma\left(r_{*}\right) P\left(r_{*}\right)
$$

As a result, for a given pressure amplitude $P\left(r_{*}\right)$ at the critical point $r_{*}$ we can uniquely determine its slope $u\left(r_{*}\right)$ and curvature $u^{\prime}\left(r_{*}\right)$, provided coefficient $\kappa \neq 1$. It is easily shown by direct computation that in the Pridmore-Brown case considered here we have $\kappa=2$ so that the last equation can be solved for $u^{\prime}\left(r_{*}\right)$.

Thus, if we have managed to arrive at $r_{*}$ by solving the initial-value problem up to the critical point and know the value of $P\left(r_{*}\right)$, then it is possible to proceed beyond the critical point by a one-step numerical method, since the solution $(P, u)$ is known and its slope $\left(P^{\prime}, u^{\prime}\right)$ is well defined at $r=r_{*}$. However, the second derivative of the solution $\left(P^{\prime \prime}, u^{\prime \prime}\right)$ does not exist at $r_{*}$, for $u^{\prime \prime}\left(r_{*}\right) \equiv P^{\prime \prime \prime}\left(r_{*}\right)$ either grows logarithmically at the critical point or is at best discontinuous, see Vilenski \& Rienstra [12]. For these reasons we use the one-step backward Euler method (27) 


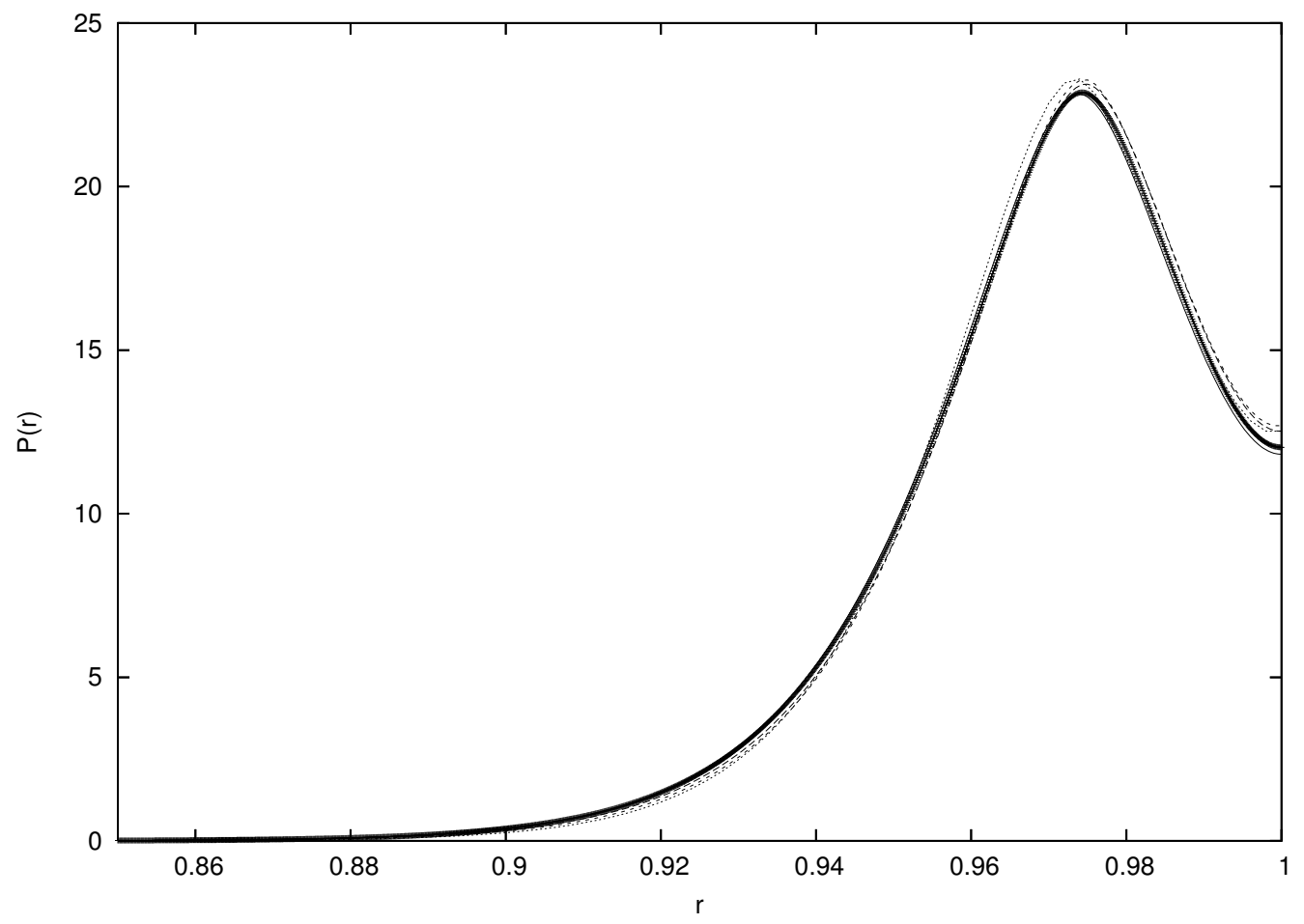

Figure 2. Convergence history of the hydrodynamic pressure eigenfunction corresponding to $\widetilde{k}=79.92, \widetilde{\omega}=25, m=15, s=h / d=0.6$ and $\bar{u}=0.3-0.5(r-1) . N=200$ dotted line, $N=400$ - short-dashed line, $N=800$ - long-dashed line, $N=1600$ - thin solid line, $N=3200$ - + symbols, $N$ is the number of mesh points.

to resolve hydrodynamic modes, rather than the higher order schemes mentioned above.

As we noted, at the critical point $r^{j+1}=r_{*}$ the coefficient $\beta^{j+1}$ goes to infinity. When this happens, formulas (27) reduce to the following two limiting relationships

$$
P^{j+1}=P^{j}, \quad u^{j+1}=0 .
$$

The second of these formulas coincides exactly with its continuous counterpart $u\left(r_{*}\right) \equiv P^{\prime}\left(r_{*}\right)=0$ needed to insure correct relationship between $u^{\prime}\left(r_{*}\right)$ and $P\left(r_{*}\right)$. Hence, the method (27) remains valid in the limit $\beta^{j+1} \rightarrow \infty$.

The ability of the proposed numerical scheme to integrate accurately across the critical layer is demonstrated in Figure 2. It illustrates the process of convergence of the numerical solution of the Pridmore-Brown equation for the hydrodynamic mode with the axial wavenumber $\widetilde{k}=79.92$, the linear mean flow profile $\bar{u}=0.3-$ $0.5(r-1)$, the hub-to-tip ratio $s=0.6$, the outer wall at $r=1$, Helmholtz number $\widetilde{\omega}=25$, azimuthal wavenumber $m=15$. In this example $0.3 \leqslant \bar{u} \leqslant 0.5$ and the hydrodynamic spectrum lies in the interval $50 \leqslant \widetilde{k} \leqslant 83.3$. Since the velocity profile is monotonous, only one critical point and one eigenmode corresponds to each hydrodynamic wavenumber $\widetilde{k}$. The plots shown in Figure 2 correspond to the discretisations with the number of mesh points $N=200,400,800,1600,3200$, 
respectively. It can be seen that all these numerical solutions are very close to each other. A better judgment about the accuracy of the method can be obtained from the comparison of the pressure predictions on the outer wall $P(1)$. Since the integration proceeded from the inner wall in the outward direction, it is the outer wall, where maximum error accumulation could be expected. For the mesh discretisations with $N=100$ (not shown in Figure 2) and $N=200,400,800,1600$ the relative errors for the value of $P(1)$ were found to be $29 \%, 4.3 \%, 5.7 \%, 4.4 \%, 1.1 \%$, with the solution for $N=3200$ taken as a reference point.

Overall, we see that the method is stable and produces reliable results for the hydrodynamic part of the spectrum, although the number of mesh points has to be kept at about 2000 in order to keep the errors below $1 \%$.

Comparison of numerical results with the exact solutions for the case of uniform mean flow showed numerical solutions to be accurate at least up to four - five decimal digits for the first several hundred eigenvalues with $N \approx 1000-3000$.

In the range of large Helmholtz numbers $\widetilde{\omega}$ and circumferential wavenumber $m$ in a non-uniform mean flow with slip the solution was tested against the WKB approximation presented in [12]. An example of such comparison for $\widetilde{\omega}=85$, $m=45, s=h / d=0.6$ and the mean flow Mach number distribution given by the parabola

$$
M=M_{\max } \frac{1+\sigma(r-s)(1-r)}{1+\sigma(1-s)^{2} / 4}
$$

with $M_{\max }=0.5$ and $\sigma=8$ (corresponding to a duct wall value of $M_{d}=$ $\left.0.76 M_{\max }\right)$ is presented in Table 1 which shows several first cut-off wavenumbers. The agreement between the WKB predictions [12] of the cut-off modes and their computed values was surprisingly good. For cut-on modes the situation is similar. The maximum error was about $6 \%$ for the wavenumber with the smallest absolute value and rapidly decreased with increasing $\widetilde{k}$ (not shown).

\section{Numerical results}

\subsection{Ducts with hard walls}

Typical plots of acoustic eigenvalues for three parabolic mean-flow velocity profiles (31) with different extents of non-uniformity $\sigma=1,8,25$ (corresponding to respective duct wall mean flow values of $M_{d}=0.96,0.76,0.50 \times M_{\max }$, or $\left.M_{d}=0.4577,0.3788,0.2758\right)$ are presented in Figure 3. Also shown is the uniform-flow spectrum $(\sigma=0)$. These plots correspond to $\widetilde{\omega}=25, m=15$, $s=h / d=0.6$. The average value of the mean flow velocity is taken to be the same and equal to 0.4596 for all $\sigma$. This is done in order to get rid of the horizontal 


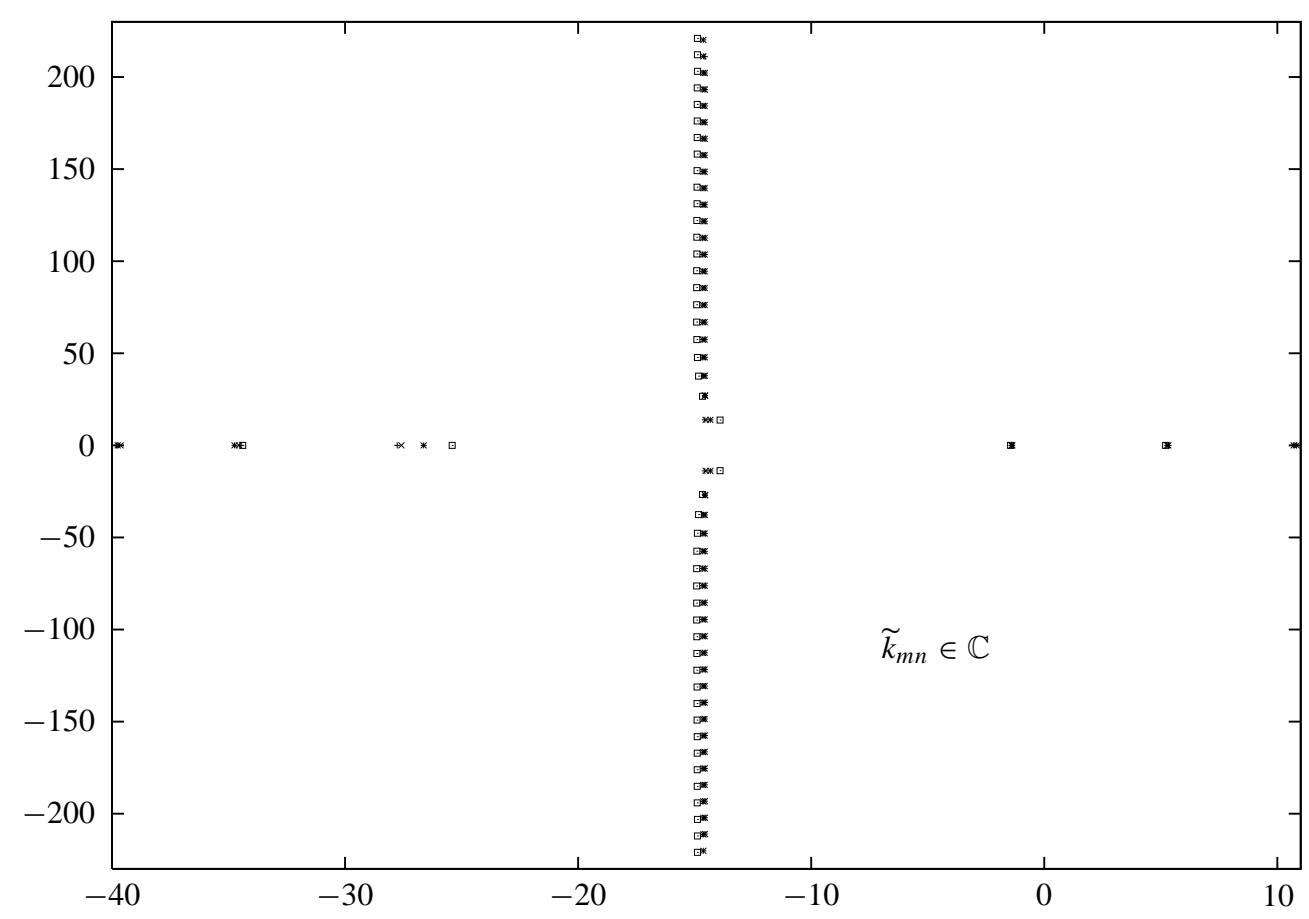

Figure 3. Effect of mean flow non-uniformity (31) on the acoustic part of axial wavenumber spectrum, $\widetilde{\omega}=25, m=15, s=h / d=0.6$. The average value of the mean flow velocity is equal to 0.4596 for all $\sigma$. Key to symbols: ' + ' is uniform flow; ' $x$ ' is $\sigma=1$ $\left(M_{d}=0.4577\right)$; ‘ $*$ ' is $\sigma=8\left(M_{d}=0.3788\right)$; ' $\square$ ' is $\sigma=25\left(M_{d}=0.2758\right)$.

shift of the eigenvalue patterns for non-zero $\sigma$ which would be present otherwise. Qualitatively all the plots are similar to the uniform-flow case. The only differences are the slight deviation of the first several complex eigenvalues from the vertical direction.

Computation of the hydrodynamic part of the spectrum for the above example proves to be more difficult. If the number of mesh points $N$ is low (say, $N<3000$ ), several real eigenvalues clustering closely to each other can be observed. Presumably, these solutions correspond to the hydrodynamic modes with the discontinuous third derivative in $r$ found in Vilenski \& Rienstra [12]. As the mesh becomes more refined the number of these singular eigenvalues rapidly decreases due to non-convergence of the Newton method for these non-smooth solutions.

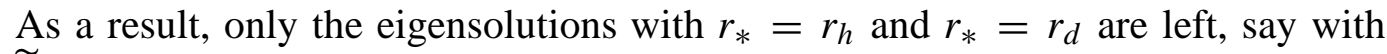
$\widetilde{k}=25 / 0.3788 \approx 66$ for $\sigma=8$.

More detailed analysis of these numerical results shows that the loss of convergence of the Newton method has the following explanation. As opposed to its continuous prototype, in the numerical problem the permissible set of hydrodynamic eigenvalues $\widetilde{k}=\widetilde{\omega} / \bar{u}\left(r_{*}\right)$ is discrete and depends on the total number of mesh points (equal to $N+1)$. When $N$ is small, the distance between these eigenvalues is large enough for the Newtonian iterations to rapidly converge to each of the related eigensolu- 


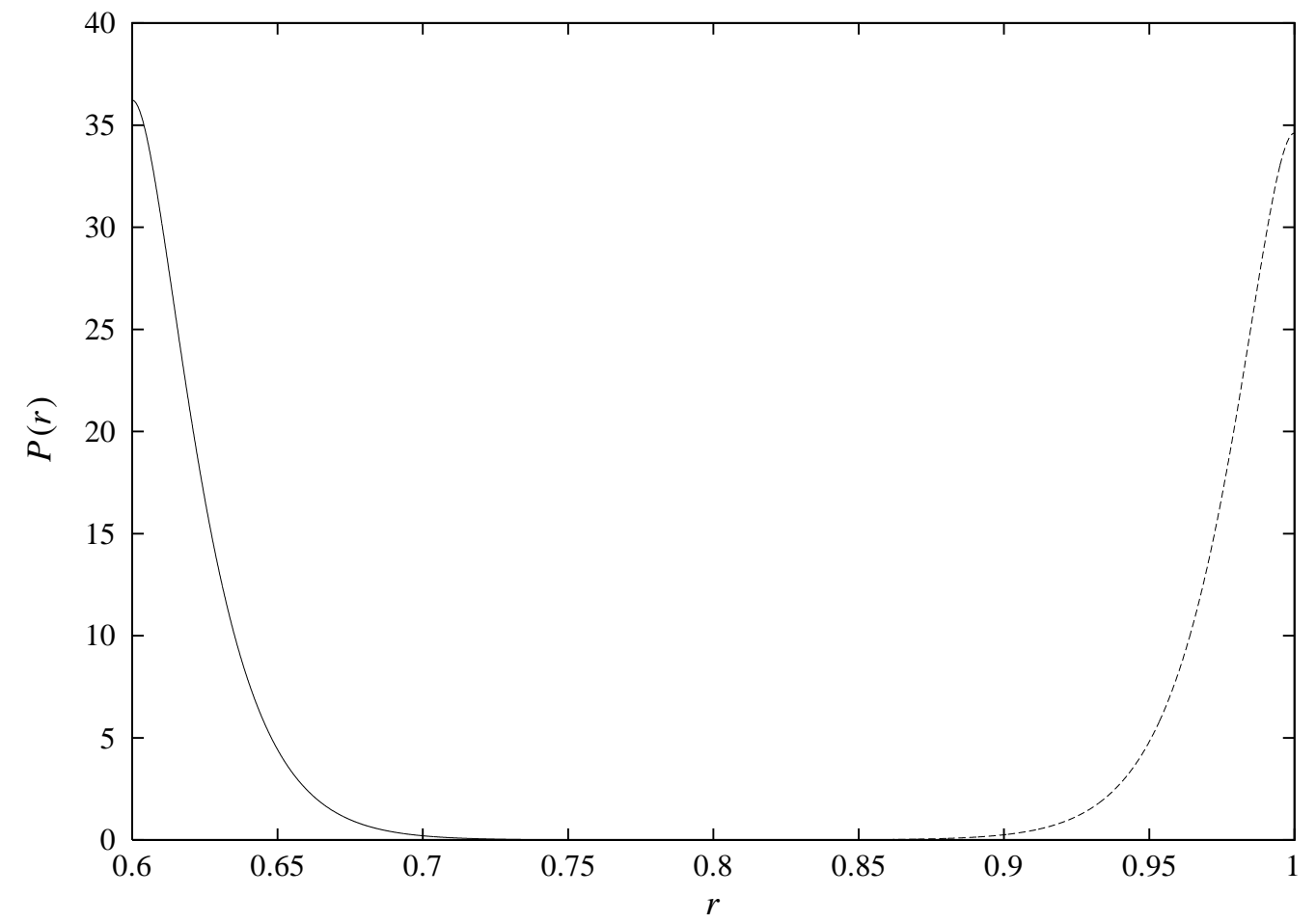

Figure 4. Two hydrodynamic pressure eigenfunctions corresponding to $\widetilde{k}=66, \widetilde{\omega}=25$, $m=15, s=h / d=0.6$ and $M_{\max }=0.5, \sigma=8$. Each eigenfunction is localized near the corresponding duct wall.

tions. However, for large $N$ hydrodynamic eigenvalues $\widetilde{k}$ lie very closely to each other. In this situation Newton's method cannot distinguish between the two neighbouring modes unless the initial prediction of $\widetilde{k}$ is specified accurately enough, in which case the convergence is restored and the lost eigensolutions are recovered. Qualitatively, the shapes of these solutions are similar to the singular mode shown in Figure 2.

For the above mentioned eigenvalue $\widetilde{k} \approx 66$ obtained for $\sigma=8$ there exist two critical points $r_{*}=r_{h}=0.6$ and $r_{*}=r_{d}=1$ with duct wall Mach numbers $M_{h}=M_{d}=0.3788$. Two eigenfunctions correspond to this eigenvalue. One of them is localized near the inner wall $r=0.6$ and the other one - near the outer wall $r=1$. In agreement with the asymptotic theory discussed in Section 2, both of these modes have smooth derivatives for $h<r<d$. They are plotted in Figure 4. Note, that, since the field equation is not symmetric with respect to the mid-radius $r=0.8$, the eigenfunctions shown in Figure 4 are also not symmetric about this point.

In the case of the multiplicity of the eigenvalue being equal to two, the eigenfunction which is localised near the outer wall can be found by imposing initial conditions (28) at the inner wall and satisfying the remaining boundary condition on the outer wall via the Newtonian iterations $(29,30)$. The other eigenfunction can be found by the same procedure, but with the roles of inner and outer wall reversed. 


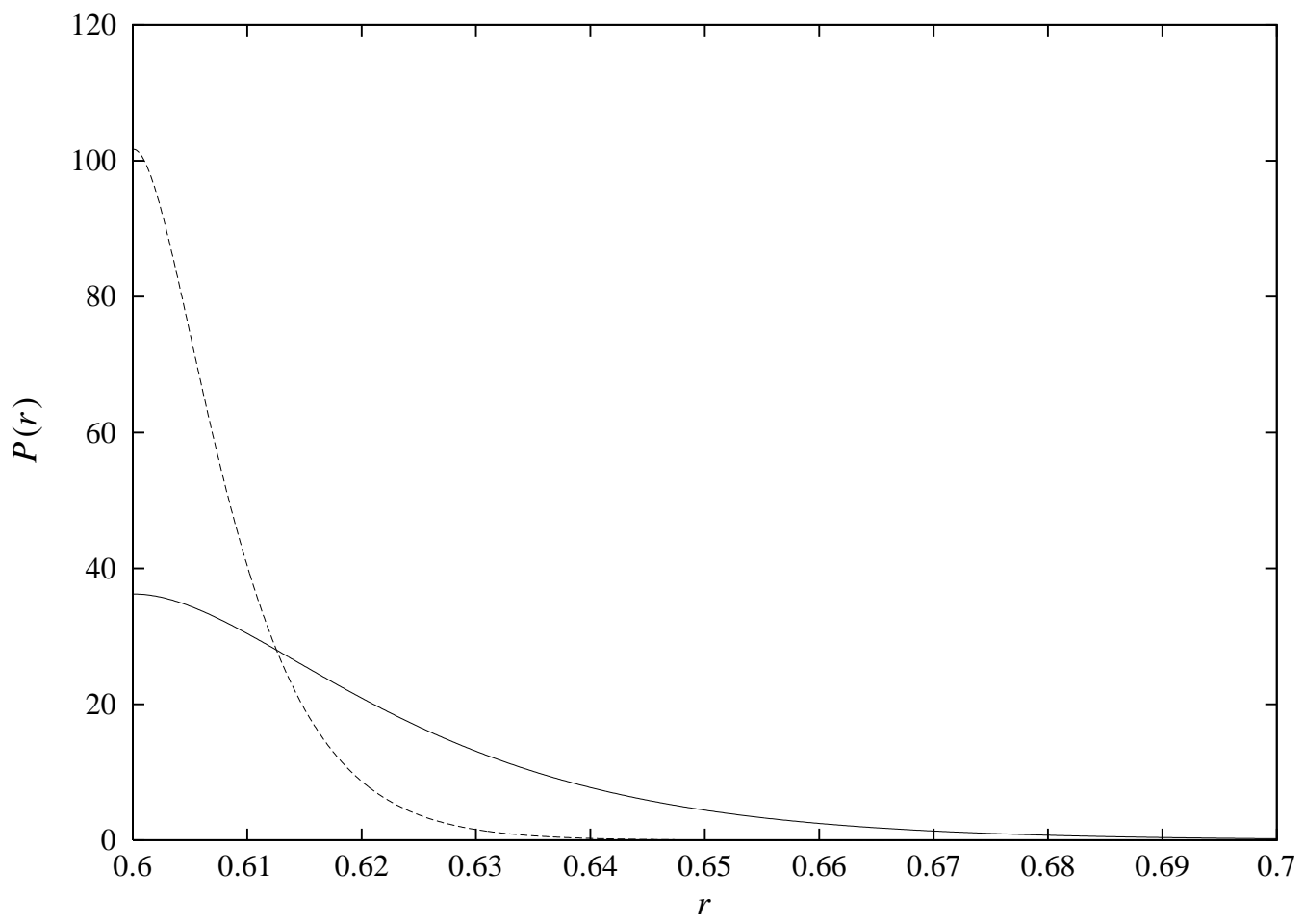

Figure 5. Hydrodynamic pressure eigenfunctions near the inner wall: $\widetilde{\omega}=25, m=15$, $s=h / d=0.6, M_{\max }=0.4596$. Wall Mach numbers are $M_{h}=0.3788$ and $M_{h}=0.1253$ for $\sigma=8$ and $\sigma=100$. The mode with the larger amplitude correspond to the smaller $M_{h}$.

If the wall Mach number is gradually decreased with the average mean flow Mach number being fixed, these hydrodynamic eigenfunctions become more compactly localized near the duct walls. This situation is illustrated in Figure 5, where two eigenmodes are shown, each corresponding to the parabolic mean-flow velocity profile (31) with $M_{\max }=0.5$ for $\sigma=8$ and $M_{\max }=0.6267$ for $\sigma=100$, respectively, corresponding to $M_{d}=0.5$ and $0.2 \times M_{\max }$, i.e., $M_{d}=0.3788$ and 0.1253. The average mean flow Mach number is equal to 0.4596 in both cases. For a mean-flow profile without near-wall non-uniformities of the boundary-layer type no new hydrodynamic modes were found numerically even for very small $M_{h}$.

\subsection{Soft-wall solutions}

This section aims to study the impact of the near-wall shear-layer gradient on the behaviour of modal solutions in a hollow duct. The following boundary-layer-type mean-flow velocity profile is taken

$$
M=M_{\max } \tanh (a(1-r)) \text { for } 0 \leqslant r \leqslant 1 .
$$

Note that $M^{\prime}(0)$ is not exactly zero but this is not a problem for the large values of $a$ that will be considered. The parameter $a>0$ controls the steepness of the mean velocity profile near the duct wall $r=1$. Apart from the limiting case of 

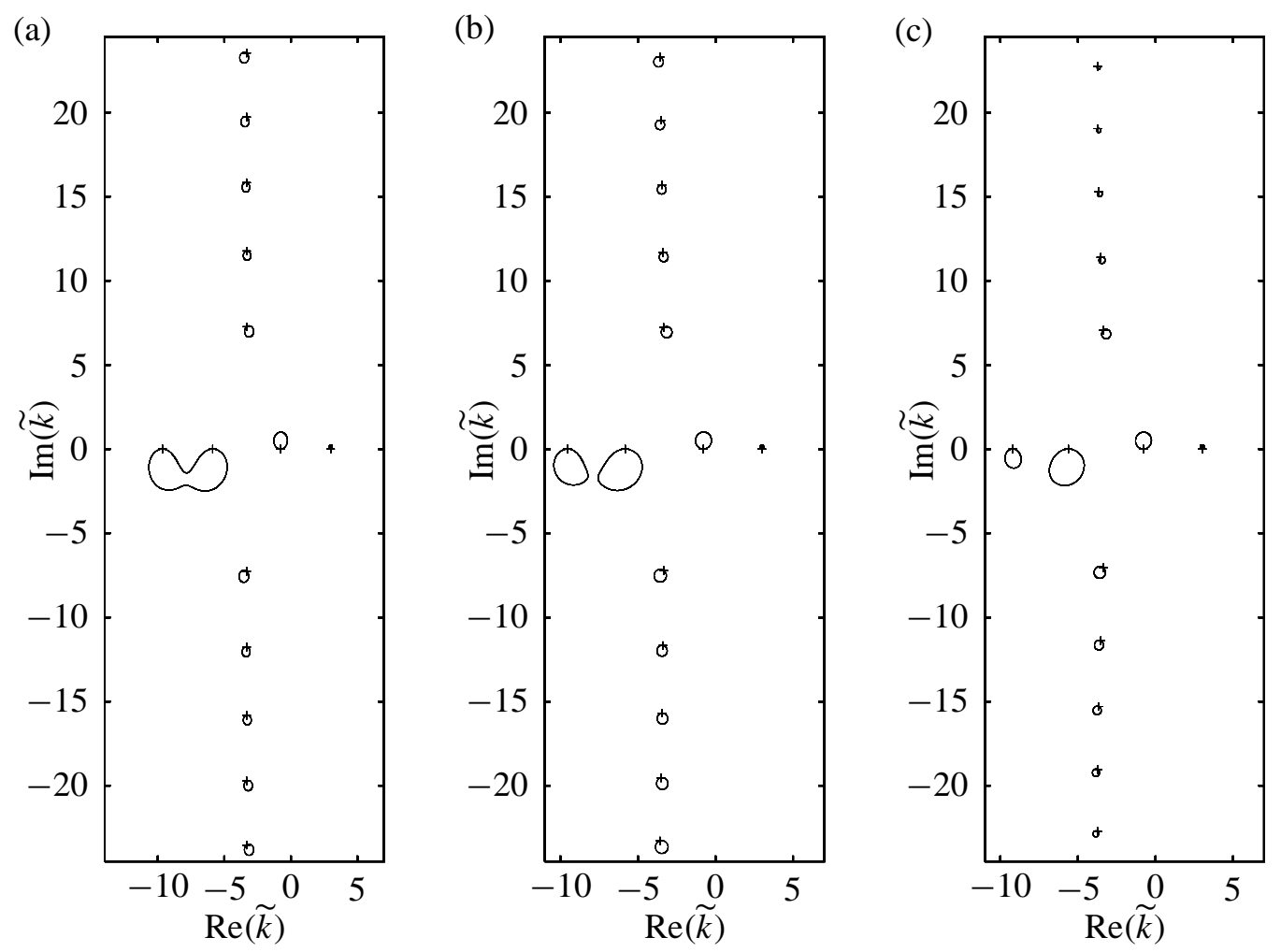

Figure 6. Trajectories of $\widetilde{k}$ for $\widetilde{\omega}=5, m=1, M_{\max }=0.5, Z_{d}=2+\mathrm{i} X,-\infty<X<\infty$. Mean flows: (a) is uniform, (b) is profile (32) with $a=250$ ( $\Delta \approx 0.011 d$ ), (c) is profile (32) with $a=50(\Delta \approx 0.053 d)$. The hard wall eigenvalues are marked with the "+" sign.

$a=+\infty$ which corresponds to the uniform mean flow, three flow situations with $a=500,250$ and 50 are considered. They correspond to the near-wall "boundary layer thickness" $\Delta$ of approximately $0.5 \%, 1.1 \%$ and $5.3 \%$ of the total duct radius, with the rest of the mean flow being virtually uniform. Here the "boundary-layer thickness" $\Delta$ is defined as the distance from the wall to the radial position $r=\Delta$, where the local velocity is equal to $99 \%$ of the "free stream velocity" $M_{\max }$. As in Rienstra [20], maximum Mach number $M_{\max }=0.5$, Helmholtz number $\widetilde{\omega}=5$ and $m=1$ are used throughout this section. The typical acoustic wavelength is thus $2 \pi d / \widetilde{\omega} \approx 1.26 d$.

Figure 6 shows typical trajectories of the axial wavenumber $\widetilde{k}$ when the reactance $X$ of the specific impedance $z_{d}$ varies from minus to plus infinity while its resistance remains fixed and relatively large $(R=2)$. The case of the uniform mean flow and two boundary-layer profiles (32) with $a=250$ and 50 are depicted. For the part of the acoustic spectrum shown in Figure 6 the quantity $\zeta_{1} \pi n / \kappa_{1} \approx 0.21 n$. Hence, with the exception of the first few indices, $n$ holds the relation $R \gg \zeta_{1} \pi n / \kappa_{1} \gg 1$. As a result, in agreement with the theoretical prediction (20), most of the axial wavenumber trajectories are close to the circles whose centres are shifted slightly to the left from their hard-wall values in the upper half-plane $\widetilde{k}$ (positive $n$ ) and to the right in the lower half-plane (negative $n$ ). As predicted by the theory, for the 
uniform mean flow and the mean velocity profile with thin near-wall shear-flow sublayers $(a=250)$ the radii of these circles slowly grow with $n$ (i.e., away from the real axis).

If the shear flow region is relatively thick, as is the case with $a=50$ in Figure $6 \mathrm{c}$, the opposite tendency prevails and the circles which are further away from the real axis have smaller radii than in the zero mean flow case studied in Rienstra [20]. The results depicted in Figure $6 \mathrm{c}$ for $a=50$, do not contradict the proposed theory, since the theory assumes that wall Mach number $M_{d}$ is non-zero. This places a restriction on the applicability of formula (20) for boundary-layer profiles.

Comparison of the wavenumber trajectories in Figures $6 \mathrm{a}, 6 \mathrm{~b}$ and $6 \mathrm{c}$ highlights the following important issue. When the boundary-layer thickness is small, the IngardMyers boundary condition can be used in combination with the "inviscid approximation" of the mean-flow profile with non-zero wall velocity (Figures 6a), instead of the actual "boundary-layer" mean-flow velocity profile which satisfies the noslip condition (Figures 6b). This is an important and well known computational feature of the Ingard-Myers boundary condition which saves the need of mesh refinement in the thin near-wall sublayer in order to resolve large mean flow gradients there. However, there arises the question about the range of validity of the approach based on the substitution of the mean flow profile with zero wall velocity by an appropriate slip-stream profile, provided that the Ingard-Myers boundary condition is imposed on the wall. Figures $6 \mathrm{a}$ and $6 \mathrm{c}$ demonstrate that although the described approach is accurate in the limit of vanishing boundary-layer thickness, the numerical results based on its application can differ perceptibly from the results obtained for a mean flow profile with vanishing wall velocity even when the boundary-layer thickness is as small as several percents of the free-field wavelength. As it will become clear from the discussion of the results shown in Figure 7 and 8, the situation gets even worse when the real part of the wall impedance becomes small. Hence, caution is needed in interpretation of the results based on slip-stream mean flow profile approximations.

Figure 7 shows the trajectories followed by the axial wavenumber $\widetilde{k}$ as $X$ varies from minus to plus infinity for $R=0.5$. Now in the uniform-flow case for almost all $n$ holds the inequality $\zeta_{1} \pi n / \kappa_{1} \gg R$. As a result, the number of circular trajectories reduces to only four and two slowly decaying vertical sinusoidal lines (21) and (22) can be observed in the upper and lower half-planes as $\operatorname{Im}(\widetilde{k}) \rightarrow \pm \infty$, respectively (Figure 7a). These lines smoothly merge with the horizontal surface-mode trajectories given by solution (23) which is valid when $\operatorname{Re}(\widetilde{k}) \rightarrow \pm \infty$. Estimates of the $\operatorname{Im}(\widetilde{k})$ for surface modes based on formulae (23) give an approximate value of \pm 8.67 which is reasonably close to the computed value of \pm 9.05 for large $\operatorname{Re}(\widetilde{k})$. However, already for the mean-flow velocity profile with a very thin near-wall sublayer shown in Figure $7 \mathrm{~b}(a=500)$ the eigenvalue pattern features substantial differences from its uniform-flow counterpart. It can be seen that only the lower surface-mode branch remains in Figure 7b. As opposed to the uniform-flow case, 

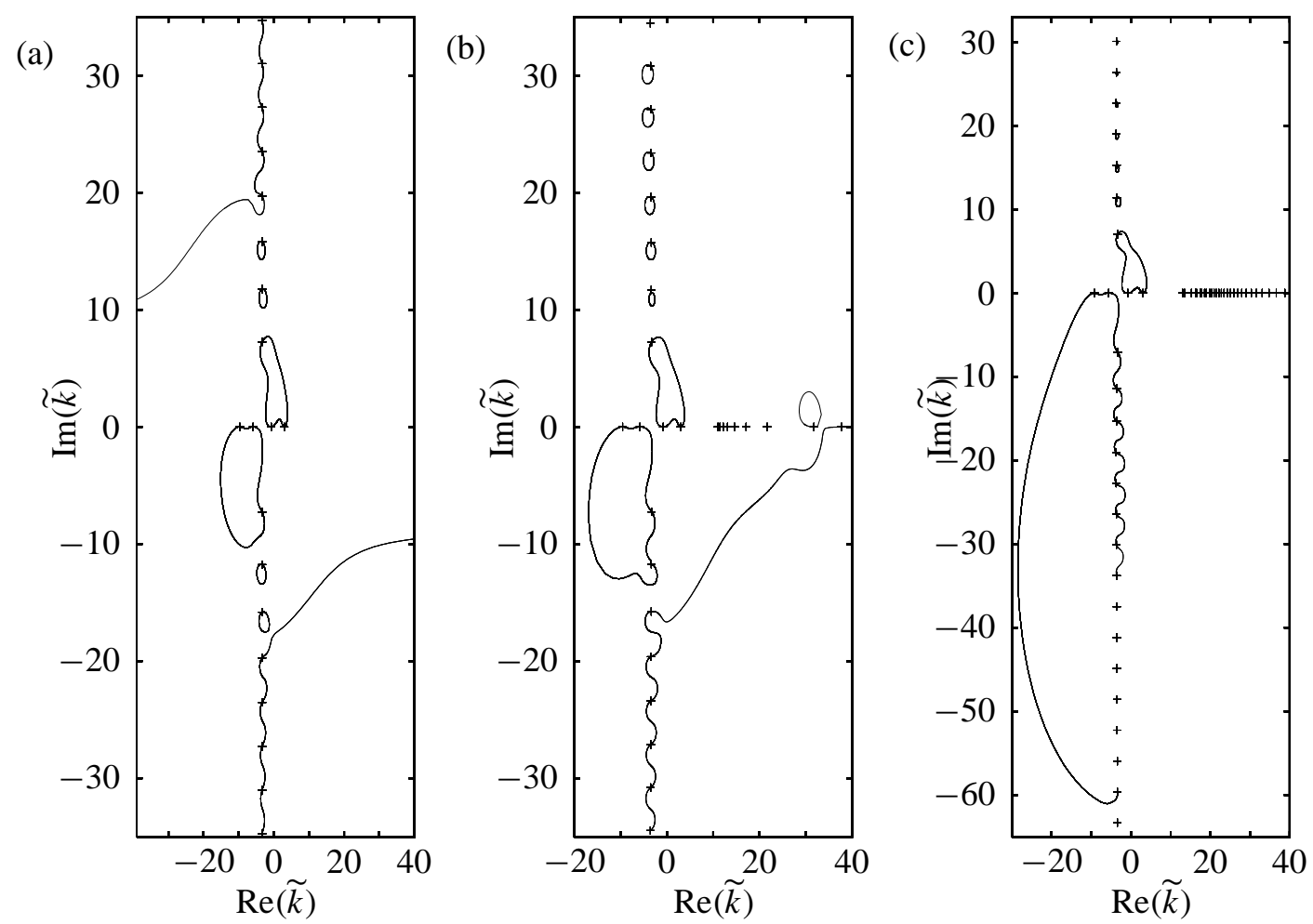

Figure 7. Trajectories of $\widetilde{k}$ for $\widetilde{\omega}=5, m=1, M_{\max }=0.5, Z_{d}=0.5+\mathrm{i} X,-\infty<X<\infty$. Mean flow: (a) is uniform, (b) is profile (32) with $a=500(\Delta \approx 0.005 d)$, (c) is profile (32) with $a=50(\Delta \approx 0.053 d)$. The hard wall eigenvalues are marked with the " + " sign.

the imaginary part of the surface-mode eigenvalue does not remain finite for large $X$ but goes to zero. In the limit $X \rightarrow \infty$ the surface mode becomes close to a hydrodynamic mode which is localized near the duct wall and convected with almost zero phase velocity. As the boundary-layer thickness increases (Figure 7c), the eigenvalue pattern deviates further away from its uniform-flow prototype and becomes structurally similar to the corresponding contour plot with zero mean-flow Mach number studied in Rienstra [20].

Computations with the profiles of the boundary-layer type reveal the appearance of real-valued hydrodynamic modes which number grows with mesh refinement. These modes can be seen in Figures $7 b, 7 c$ and also in Figure 8. Since it has been shown already that the proposed numerical scheme is able to integrate across the critical layer and gives convergent solutions for hydrodynamic modes, the hydrodynamic modes presented in Figures 7 and 8 are a genuine feature of the PridmoreBrown equation and not a shortcoming of the numerical method. The reason why the number of numerically found hydrodynamic modes must grow with mesh refinement is as follows. The axial wavenumbers $\tilde{k}$ which correspond to hydrodynamic modes of the Pridmore-Brown equation are given by the relationship $\widetilde{\omega}-$ $\widetilde{k} \bar{u}\left(r_{*}\right)=0$. As $r_{*}$ varies continuously between the inner and the outer walls, $\tilde{k}$ changes continuously in the interval $\widetilde{\omega} / \bar{u}_{\max } \leqslant \widetilde{k} \leqslant \widetilde{\omega} / \bar{u}_{\min }$. However, if the in- 

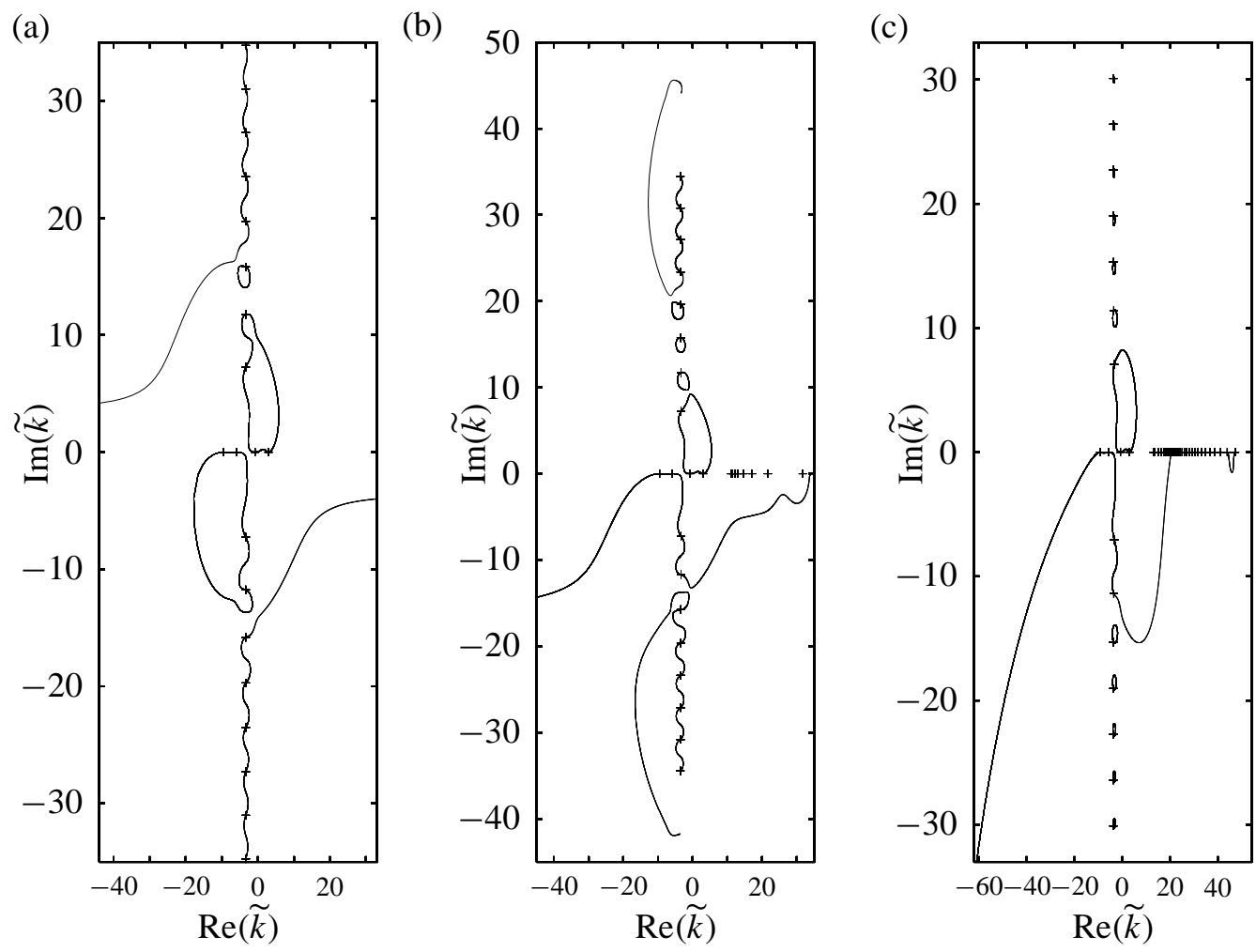

Figure 8. Trajectories of $\widetilde{k}$ for $\widetilde{\omega}=5, m=1, M_{\max }=0.5, Z_{d}=0.2+\mathrm{i} X,-\infty<X<\infty$. Mean flow: (a) is uniform, (b) is profile (32) with $a=500(\Delta \approx 0.005 d)$, (c) is profile (32) with $a=50(\Delta \approx 0.053 d)$. The hard wall eigenvalues are marked with the "+" sign.

dependent variable $r$ is allowed to vary only over a discrete set of $N+1$ points $r^{j+1}=s+j \delta, j=0,1,2,3 \ldots N$, the equation for $\widetilde{k}$ can also have only a finite set of discrete solutions. Its total number is controlled by the mesh discretisation parameter $N$. As the number of mesh points grows, so does the number of critical points and, as a result, the number of associated eigenvalues $\widetilde{k}\left(r_{*}\right)$.

Although further decrease of the real part of the wall impedance $R$ has little effect on axial wavenumber contours for the uniform flow, the trajectories of $\widetilde{k}$ for the boundary-layer mean flow profiles undergo substantial structural changes as $R$ is reduced from 0.5 to 0.2 (see Figure 8). One remarkable feature of the eigenvalue patterns obtained for the boundary-layer type mean flow profiles and low $R$ is that as the imaginary part of the wall impedance $X$ varies from minus to plus infinity one of the lower half-plane cut-off acoustic modes gradually evolves into a hydrodynamic mode.

The hydrodynamic modes obtained for rigid walls move off the real axis if the imaginary part of the wall impedance is allowed to vary from minus to plus infinity. Our computation shows that for $a=50$ ("thick boundary layer") these modes are shifted below the real axis, whereas for $a=500$ ("thin boundary layer") they are shifted into the upper half plane. Two typical trajectories of such modes are shown in Figure $7 \mathrm{~b}$ and Figure 8c for $a=500$ and $a=50$, respectively. In the 
former case the modal wavenumber $\tilde{k}$ follows a circular trajectory and returns to its starting value. In the latter case the trajectory is not closed and the limiting values of $\widetilde{k}$ for $X=+\infty$ and $X=-\infty$ differ. Variation of resistance $R$ in the range $0.2 \leqslant R \leqslant 2.0$ for fixed $X$ and $a$ did not result in the change of the orientation of the hydrodynamic spectrum with respect to the real axis.

If both former modes are to be interpreted as right-running, it would imply that the mode is unstable for the thicker boundary layer and stable for the thinner boundary layer. Although speculative (we don't know if we can speak of the same mode), it is worth noting that this is opposite to the trends reported by Michalke [26,27] for stability of free shear layers. Here a thicker shear layer stabilizes the perturbation. Further study of stability and causality issues for these modes is needed.

\section{Conclusions}

A robust numerical algorithm for determination of duct eigenmodes in sheared mean flows was developed. For sheared mean flows it was tested against existing data and was shown to be capable of handling very high frequencies.

Existing analytical theory based on the WKB approach was found to be in excellent qualitative agreement with the numerical study which also made it possible to bridge the gap in the qualitative behaviour of the solution, in the cases where the theoretical data was not available.

The obtained results show that for the mean flow profiles with non-zero wall Mach numbers there are two hydrodynamic eigenmodes (one for each wall) which are smooth inside the flow region, but have a critical point on the wall. Each of these modes is localized near the corresponding wall of the duct and rapidly decay as the radial distance from the wall increases. Apart from these two, there is a continuum of hydrodynamic modes with the critical point inside the flow region (Figure 2). These modes are singular inside the flow region, and their pressure amplitude has only two continuous derivatives at the critical point.

For the boundary-layer mean-flow profiles which satisfy the no-slip condition our numerical analysis suggests the possibility of the existence of the continuous unbounded hydrodynamic spectrum. This spectrum is real-valued for rigid wall boundary conditions and is shifted into the upper or the lower part of the complex wavenumber plane when the imaginary part of the wall impedance varies from minus to plus infinity with its real part being fixed. Preliminary computations suggest that the direction of this shift is controlled by the properties of the mean flow velocity profile. However, further analysis is needed. 


\section{Acknowledgements}

This work was supported by the "Messiaen" European collaborative project (EU Technical Officer Dietrich Knörzer and Coordinator Jean-Louis Migeot, Free Field Technologies).

\section{References}

[1] Pridmore-Brown, D. C., 1958, Sound Propagation in a Fluid Flowing through an attenuating Duct, Journal of Fluid Mechanics, vol. 4, pp.393-406.

[2] Zorumski,W.E., 1974, Acoustic theory of axisymmetric multi-sectioned ducts. NASA TR R-419.

[3] Rienstra, S.W., 1999, Sound transmission in slowly varying circular and annular lined ducts with flow, Journal of Fluid Mechanics, vol. 380, pp.279-296

[4] Rienstra, S.W., 2003, Sound propagation in slowly varying lined flow ducts of arbitrary cross-section, Journal of Fluid Mechanics, vol. 495, pp.157-173

[5] Cooper, A.J., Peake, N., 2001, Propagation of unsteady disturbances in slowly varying duct with swirling mean flow. Journal of Fluid Mechanics, vol. 445, pp.207-234.

[6] Eversman, W. 1991, Theoretical model for duct acoustic propagation and radiation. In Aeroacoustics of Flight Vehicles: Theory and Practice. Volume 2: Noise Control (Ed. H.H. Hubbard), chapter 13, pp.101-163.

[7] Golubev, V.V, Atassi, H.M., 1998, Acoustic vorticity waves in swirling flows. Journal of Sound and Vibration, vol. 209(2), pp.203-222.

[8] Tam, C.K.W., Auriault, L., 1998, The wave modes in ducted swirling flows. Journal of Fluid Mechanics, vol. 371, pp.1-20.

[9] Kousen, K.A. 1999, Eigenmodes of Ducted Flows With Radially-Dependent Axial and Swirl Velocity Components, NASA /CR-1999-208881.

[10] Nijboer, R. 2001, Eigenvalues and eigenfunctions of ducted swirling flows. AIAA 2001-2178, pp.1-11. 7th AIAA/CEAS Aeroacoustics Conference Maastricht, 28-30 May 2001,

[11] Cooper, A.J., Peake, N., 2005, Upstream-radiated rotor-stator interaction noise in mean swirling flow. Journal of Fluid Mechanics, vol. 523, pp.219-250.

[12] Vilenski, G. \& Rienstra, S.W. 2006, Acoustic modes in ducted shear flow. Accepted for publication in the Journal of Fluid Mechanics.

[13] Envia, E., 1998, A High Frequency Model of Cascade Noise, AIAA-98-2318, 4th AIAA/CEAS Aeroacoustics Conference, Toulouse, France, June 2-4, 1998. 
[14] Rienstra, S.W., Tester, B.J., 2005, An Analytic Green's Function for a Lined Circular Duct Containing Uniform Mean Flow, AIAA 2005-3020 of the 11th AIAA/CEAS Aeroacoustics Conference, Monterey, CA, 23-25 May 2005. Revised version submitted to Journal of Sound and Vibration.

[15] Rienstra, S.W., 2006, Impedance Models in Time Domain, including the Extended Helmholtz Resonator Model, AIAA 2006-2686, 12th AIAA/CEAS Aeroacoustics Conference, 8-10 May 2006, Cambridge, MA, USA

[16] Rienstra, S.W., 2007, Acoustic Scattering at Hard-Soft Transition with Flow. Accepted for publication in Journal of Engineering Mathematics as the 2007 Lighthill Memorial Paper.

[17] Brambley, E.J., Peake, N., 2006, Surface-waves, Stability, and Scattering for a Lined Duct with Flow, AIAA 2006-2688, 12th AIAA/CEAS Aeroacoustics Conference, 810 May 2006, Cambridge, MA, USA

[18] Ingard, K.U., 1959, Influence of fluid motion past a plane boundary on sound reflection, absorption and transmission. Journal of the Acoustical Society of America. vol. 31(7), pp.1035-1036.

[19] Myers, M.K., 1980, On the acoustic boundary condition in the presence of flow. Journal of Sound and Vibration. 71(3), pp. 429-434.

[20] Rienstra, S.W. 2003, A classification of duct modes based on surface waves. Wave Motion. vol. 37 pp.119-135.

[21] Miranker, W.L., 1981 Numerical methods for stiff equations and singular perturbation problems. D. Reidel Publishing Company.

[22] Butcher, J.C., 2003, Numerical methods for ordinary differential equations. Wiley.

[23] Fornberg,B., Driscoll, T.A., 1999 A fast spectral algorithm for nonlinear wave equations with linear dispersion. Journal of Computational Physics, vol. 155, pp.456467.

[24] Chan, T. F. \& Kerkhoven, T. 1985 Fourier methods with extended stability intervals for the Korteweg-de Vries equation, SIAM J. Numer. Anal. 22, 441.

[25] Deuflhard, P., Bornemann, F., 2000 Scientific computing with ordinary differential equations. Springer.

[26] Michalke, A., 1965, On Spatially Growing Disturbances in an Inviscid Shear Layer, Journal of Fluid Mechanics, vol. 23(3), pp. 521-544.

[27] Michalke, A., 1984, Survey On Jet Instability Theory, Prog. Aerospace Sci., vol. 21, pp. 159-199. 